\title{
Technology of Point Focusing of Electromagnetic Waves (Spotforming)
}

\author{
A. Chugunov, R. Kulikov, N. Petukhov, I. Indrikov \\ R\&D center of Radio \& Electronics Department of MPEI, \\ National Research University MPEI, \\ Moscow, Russian Federation \\ E-mail: coolicoff@gmail.com
}

\begin{abstract}
The article considers the technology of point focusing of electromagnetic waves, which allows to transmit electromagnetic energy to consumer more effectively than beamforming. The technology is based on the principle of operation of antenna arrays, but with the distribution of dipole antennas around the perimeter of the area under consideration, which makes it possible to obtain an increase in the power of the electromagnetic field only at one point in this area, and not in the beam. The article presents the results of theoretical studies as well as mathematical modeling that confirm the feasibility and describe the properties and characteristics of spotforming technology. The experimentally obtained characteristics of the generated electromagnetic fields measured by the real prototype are shown. Spotforming is technology of next-generation spatial filtering systems that can be used in high-speed data transmission systems and 5th generation mobile networks.
\end{abstract}

Keywords: phased arrays, microwave antenna arrays, transmitting antennas, electromagnetic fields, $5 \mathrm{G}$ mobile networks, microwave interference, wireless communication, wireless power transmission.

\section{INTRODUCTION}

The basis of spatial processing technologies is the use of separate directional antennas and antenna arrays (AA) of weakly directional elements - beamforming, MIMO (multiple input - multiple output) [3, 7]. The advantage of directional antennas is simplicity. The advantage of antenna arrays is the ability to quickly change the radiation pattern through electronic control of the channel phases and due to the absence of moving parts. These approaches provide forming a narrow beam of the radiation pattern [4].

In the case of directed radiation, electromagnetic energy is concentrated along the line of sight transmitter-receiver. It allows to reduce energy losses compared to non-directional radiation. At the same time, the receiver is only at one point of the line of sight, and in fact, high electromagnetic field strength is required to be provided only at the receiver location (more precisely, the receiver's antenna) [3].

This approach, which we called "spotforming", makes the use of electromagnetic energy even more efficient than beamforming (Fig. 1).

(C) Chugunov A., Kulikov R., Petukhov N., Indrikov I., 2018 


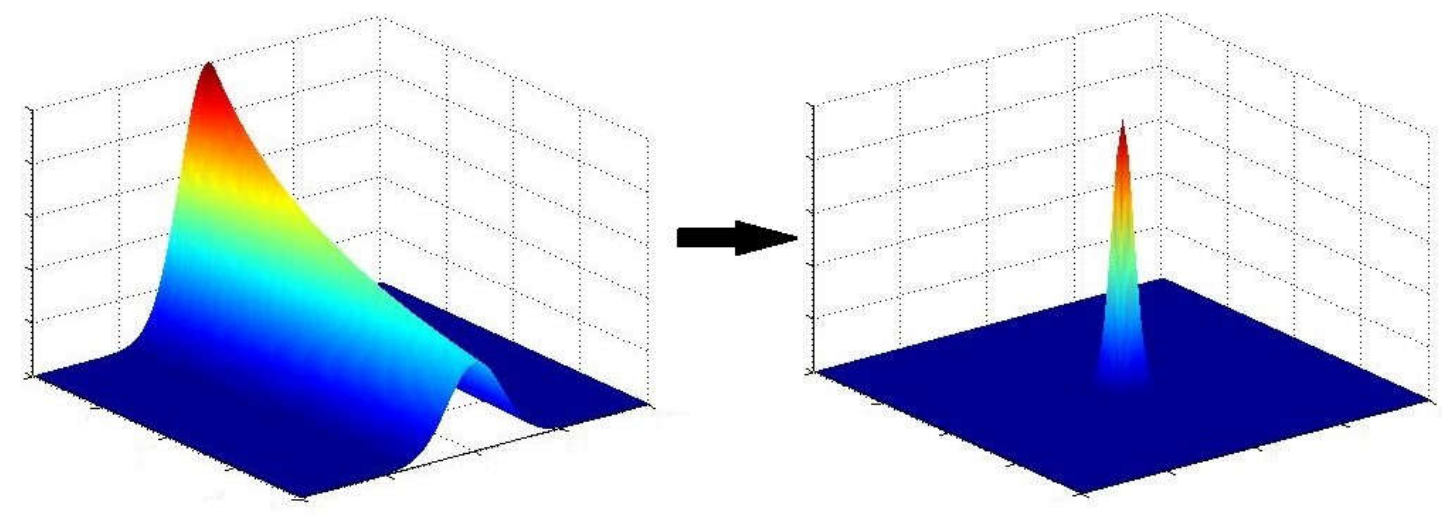

Figure 1. The transition from beamforming to spotforming: $\mathrm{z}$-axis means power of electromagnetic field, $\mathrm{x}, \mathrm{y}$-axes mean planar coordinates

\section{SPOTFORMING}

Spotforming technology or the technology of point focusing of electromagnetic waves implies the use of an antenna system similar to AA. The difference from the known AA is the installation of elements of AA not in one place, but in the space around the working area (room), in which there is need to form a local peak of the field.

In the article [5] it is suggested to use a set of radiators installed along the perimeter around a given point and spatially separated at a distance of several kilometers or more, with several generators synchronized in time. Our approach is intended for the use in working areas, the size of which ranges from a few meters to tens of meters with a single generator and control of the phases of the dipoles.

Antenna arrays as systems with a controlled radiation pattern of a complex shape, is based on a large number of simple weakly directional radiators. Symmetrical short antennas (Dipole Antenna) act as such radiators. Dipole antenna is a short thin piece of the conductor. The characteristics of symmetrical antennas are more fully described by the model of an elementary electric radiator [1]. The case where the length of the conductor is equal to half the wavelength is of greatest interest. Such an antenna is called a half-wave vibrator, whose arm length is $\lambda / 4$. Its radiation pattern has the form of a torus (Fig. 2), this means that maximum radiation of such an antenna is directed perpendicular to its axis, and there is no radiation along the axis.

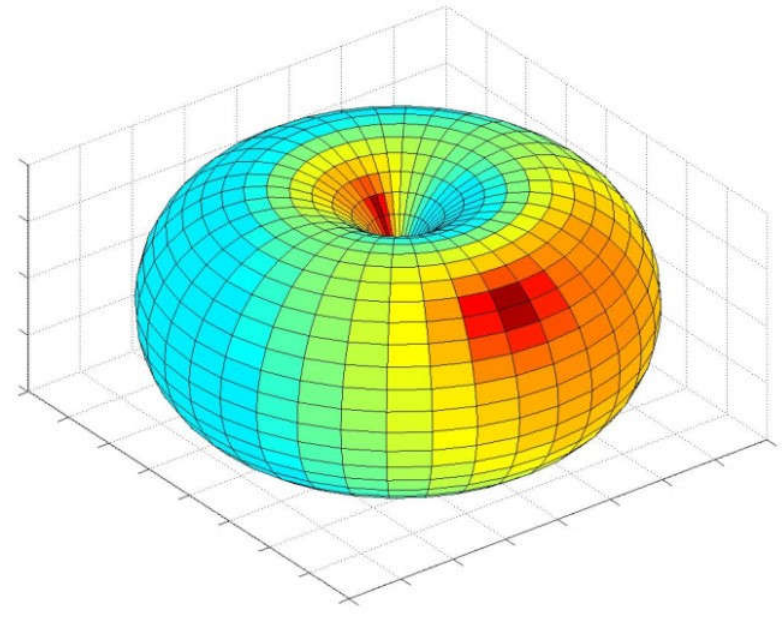

Figure 2. The radiation pattern of a half-wave vibrator 
It can be assumed that the electromagnetic wave has a flat front in the far field and contains mutually perpendicular components of electric and magnetic field intensities lying in a plane perpendicular to the direction of wave propagation. With the vertical position of the dipole, the vector of the electric field intensity of the electromagnetic field in the far zone has only the vertical component and is described as follows [2]:

$$
E_{\theta}(r, t)=-i \frac{I_{0} Z_{C}}{2 \pi} \sin \theta \frac{e^{-i(\omega t-k r)}}{r} e^{-i \varphi_{0}},
$$

where $I_{0}$ - current in the arms of a dipole; $Z_{C}$ - wave impendance; $k$-wave number; $\lambda$ - wave length; $\varphi_{0}$ - initial phase of electric current; $\theta$ - angle measured from the dipole axis; $r$ - the distance between the dipole and the point in which the tension is calculated; $i$ - imaginary unit.

Let us fix the moment of time. The amplitude of the electric field intensity vector decreases inversely proportional to the distance, and the phase increases linearly in proportion to the distance from the dipole. Approximately, this can be written as follows:

$$
E_{\theta}(r) \sim \frac{e^{-i\left(-k r+\varphi_{0}\right)}}{r}
$$

If the initial phase of the current in the conductor is known, the phase $\Phi$ of the electromagnetic wave at a given distance $\tilde{r}$ from the dipole calculated by the formula:

$$
\Phi_{\bar{r}}=-k \tilde{r}+\varphi_{0} .
$$

Consider the case when there are $N$ half-wave antennas with known coordinates. The antennas are located around the working area (Fig. 3). All antennas are connected to the same generator via phase shifters. According to the formula (2), the field of the $i$-th radiator:

$$
E_{i}(r) \sim \frac{e^{-i\left(-k r+\varphi_{i}\right)}}{r} .
$$

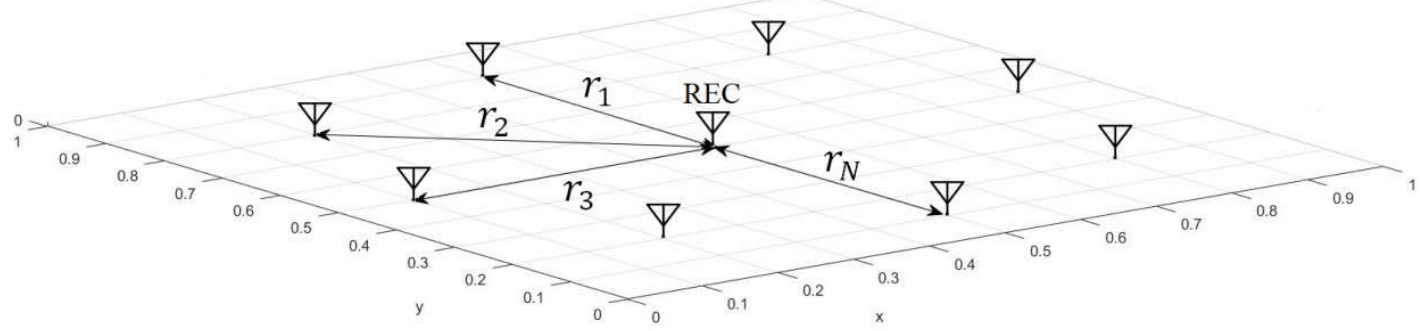

Figure 3. Illustration of the point interference of waves of a large number of antennas

We specify a point within the working area with known coordinates and form a local peak of the electric field in it. Receiver's antenna (REC) is placed in that point. The initial phases $\varphi_{i}$ are set with phase shifters in such a way that at a given point the phases of all individual electromagnetic waves coming from the dipoles are equal.

For example, for certainty, at a fixed point in time:

$$
\Phi_{i}=-k r_{i}+\varphi_{i}=0,
$$


where $r_{i}$ is the distance between the given point and the $i$-th dipole antenna. We assume that $r_{i}>>\lambda$ (the far field for all dipoles). This condition ensures the in-phase of the electromagnetic waves of all dipoles at a given point. The condition of in-phase in general form:

$$
\Phi_{i}=-k r_{i}+\varphi_{i}=\Phi \pm 2 \pi k, k=0,1,2, \ldots
$$

Under condition (6), the in-phase addition of electromagnetic waves occurs at a given point. The module of total electromagnetic field intensity:

$$
\left|E_{\Sigma}\right|=\sum_{i=1}^{N}\left|E_{i}\right|
$$

where $\left|E_{i}\right|$ - the electric field intensity, formed by the i-th dipole antenna at a given point.

In the central part of the working area, the distances from all the antennas to the given point are approximately equal $r_{1} \approx r_{2} \approx \ldots \approx r_{N}$. Then the modules of electric field intensity of each dipole antenna at this point are approximately equal to $\left|E_{1}\right| \approx\left|E_{2}\right| \approx \ldots \approx\left|E_{N}\right|=\left|E_{d i p}\right|$. So you can write:

$$
\left|E_{\Sigma}\right|_{\max } \sim N\left|E_{d i p}\right|
$$

where $\left|E_{d i p}\right|$ - the electric field intensity formed by the single dipole antenna at a given point.

The module of electric field intensity at a given point in the central part of the working area is proportional to the number of dipole antennas and the current amplitude on a single dipole.

Here and further, the point where the in-phase addition of waves occurs is called the point of maximum, or maximum, and all other points are called points of periphery, or periphery. The values of the intensities and powers of the electromagnetic field at such points will be denoted by the corresponding indices $E_{\max }$ or $E_{p e r}\left(P_{\max }\right.$ or $\left.P_{p e r}\right)$.

In all the rest of the working area, except for a given point, the condition (6) is violated and the addition of $N$ electromagnetic waves occurs with random phases:

$$
\left|E_{\Sigma}\right|_{p e r}=\left|\sum_{i=1}^{N}\right| E_{i}\left|e^{-j \Phi_{i}}\right|,
$$

where $\Phi_{i}$ is a random phase which has a uniform distribution on the interval $[-\pi, \pi]$. The module of the resulting electric field intensity in the periphery is proportional to the root of the number of dipoles and the current amplitude of the dipoles on the average.

$$
\left|E_{\Sigma}\right|_{p e r} \sim \sqrt{N}\left|E_{\text {dip }}\right| \text {. }
$$

The electromagnetic field intensity of the periphery increases more slowly than this value at the maximum with increasing the number of dipoles. This is an important feature of a distributed system of phase controlled antennas, and allows to form an electric field at a given point. This is due to in-phase focusing of radio waves at a given point by controlling the phase of each dipole.

The power of the electromagnetic field is proportional to the square of the electric field intensity. Therefore:

$$
\frac{P_{\max }}{P_{p e r}}=\left(\frac{\left|E_{\Sigma}\right|_{\max }}{\left|E_{\Sigma}\right|_{p e r}}\right)^{2} \sim\left(\frac{N\left|E_{d i p}\right|}{\sqrt{N}\left|E_{d i p}\right|}\right)^{2}=N .
$$


The ratio of the power at the point of maximum to the power of the periphery is proportional to the number of dipoles. Figure 4 shows the relationship between the ratio of the power of the maximum to the power of the periphery averaged over the three-dimensional space of the working zone, and the number of dipoles used, obtained by modeling in the presence of single and double reflections of radio waves from walls, ceiling and floor. That results were obtained by simulation in Matlab. Simulation conditions: operating frequency $f=2 \mathrm{GHz}$, the area under consideration is a parallelepiped size of $8 \times 4 \times 3 \mathrm{~m}$, distribution of dipoles along the walls is uniform, the dielectric constant of the reflecting material is $\varepsilon=5$, the maximum point coordinates are $[3.55,3.08,1.74] \mathrm{m}$ (asymmetric location of the point of maximum).

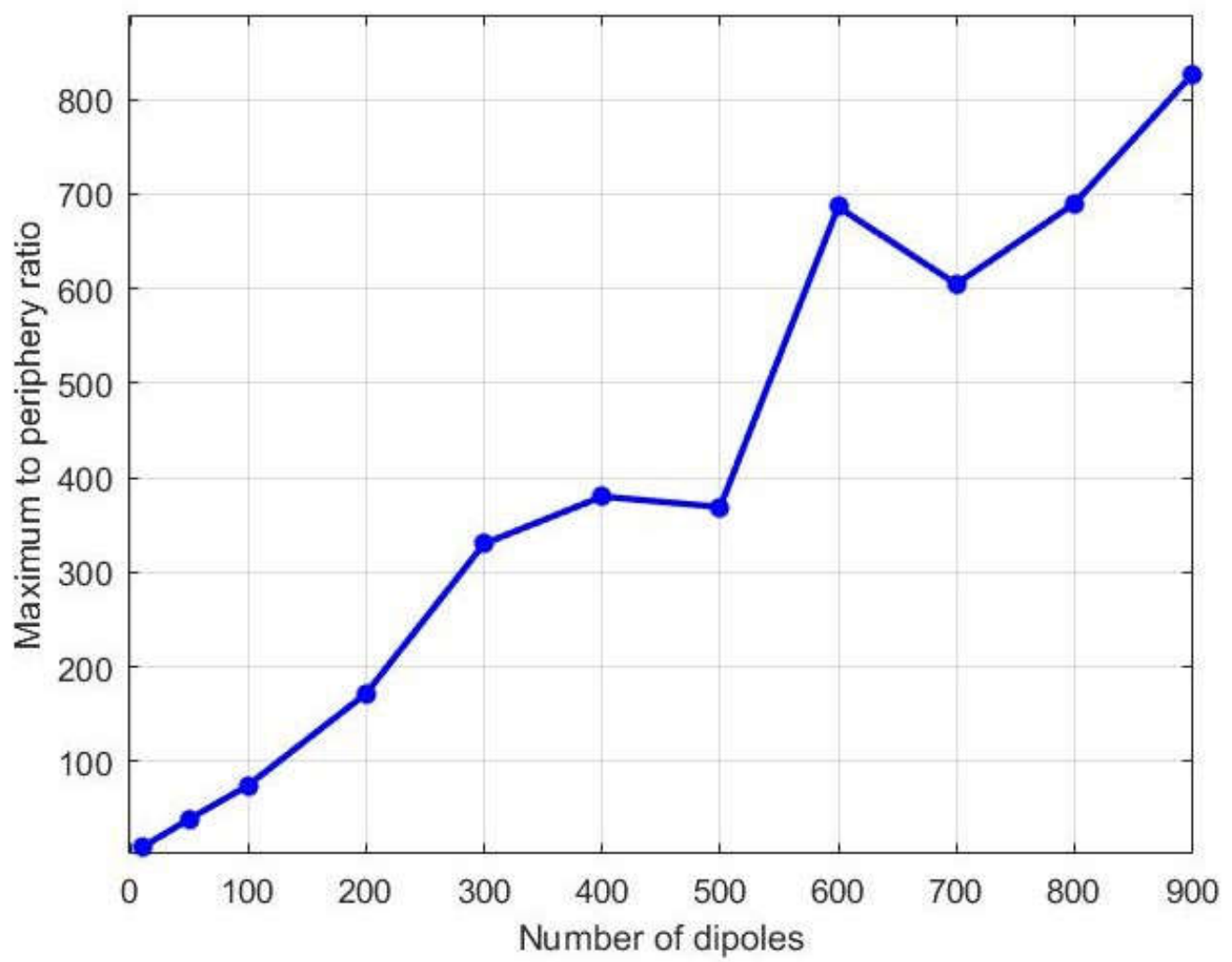

Figure 4. The estimated relationship between the ratio of the power of the maximum to the power of the periphery and the number of dipoles taking into account the effect of reflection from the walls

The above relations (8), (10), (11) are approximate. In reality, as shown in the figure, in addition to direct propagation of radio waves in buildings, reflections from walls and objects in the work area play an important role. It is furthermore necessary to take into account the mutual influence of the dipoles $[1,2]$. Strictly taking into account this influence in the general case is not possible. The following sections describe a prototype for verifying the characteristics of an approach in practice.

\section{PROTOTYPE}

For experimental confirmation of the feasibility of spotforming technology and researching of its properties, a prototype was developed, which is a model of a room size of $1 \times 1 \times 1 \mathrm{~m}$. On the walls of the room there are dipoles, which are half-wave vibrators. In order to comply with the interference condition, - coherence of radio waves, all dipole antennas are connected to one monochromatic signal generator via system of power dividers. For in- 
vestigation the electromagnetic field formed in the area under consideration, is used receiver, which is also a half-wave vibrator. It is connected to a spectrum analyzer to measure the power of the electromagnetic field at the receiver's location. The receiver is moved by means of special mechanisms, which makes it possible to analyze the generated electromagnetic field pattern entirely in any cross section. The prototype and its functional diagram are depicted in Fig. 5 and Fig. 6, respectively.

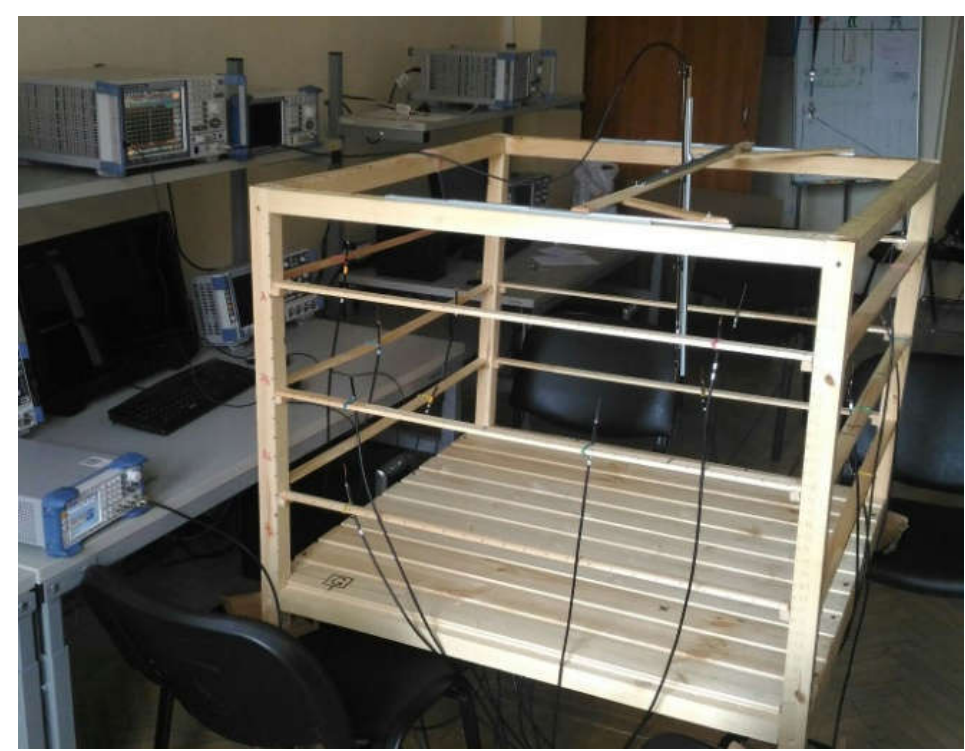

Figure 5. The prototype of spotforming technology

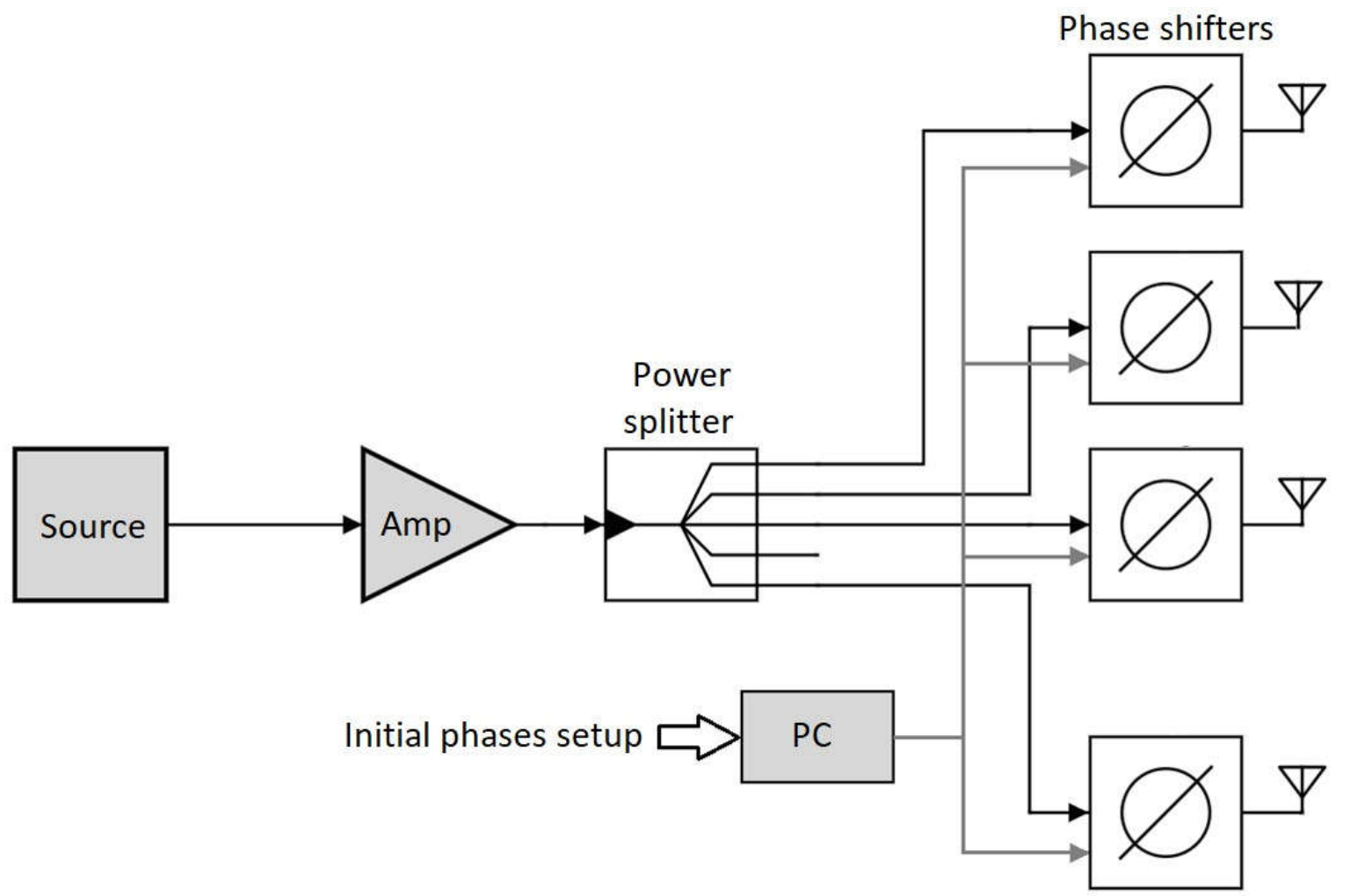

Figure 6. The block diagram of the spotforming system's transmitting part 
The block diagram of the prototype for an arbitrary number of dipole antennas includes a signal generator, power dividers, digital phase shifters that set the initial phase of each dipole antenna to focus the radio waves at a given point. Their number is equal to the number of dipole antennas used. A computer carries out the control of phase shifters.

\section{EXPERIMENTS}

The prototype allowed us to observe the real field patterns, formed by a system of distributed dipole antennas. Figure $7(a, b)$ shows the obtained power distribution of the electromagnetic field using 8 and 16 dipole antennas. Formed electromagnetic fields are characterized by a clear spot peak, but also by the presence of subordinate maximum, which is inferior in magnitude to the main one. Measurement of the field pattern is carried out in a horizontal plane passing through the maximum point, and measured area located in the center of the "room" and $20 \mathrm{~cm}$ apart from the walls (see Fig. 7). This area corresponds to the far field for all dipole antennas. This fact allows us to assume the front of the electromagnetic wave to be flat and ensures the fulfillment of the relations (1-11). The dipole antennas are located at two different heights to avoid symmetry and the occurrence of subordinate maximum.

The working frequency of the experiment is $2 \mathrm{GHz}$. The measurements were carried out with a step several times smaller than the wavelength. For the experiment using eight dipole antennas, the grid spacing is $4 \mathrm{~cm}$, for the experiment using sixteen dipole antennas $-2 \mathrm{~cm}$. It should be noted that in the first experiment the point of maximum is located approximately in the center of the area under study (its coordinates are $x=54 \mathrm{~cm}, y=50 \mathrm{~cm}$ ), and in the second - closer to its edge (its coordinates are $x=26 \mathrm{~cm}, y=70 \mathrm{~cm}$ ). This is done to show that the focusing of waves is attainable at any point of the area under study.

If a small number of dipole antennas are used, the field pattern will be characterized by subordinate maximum of power commensurate with the power of the point of maximum. As the number of dipole antennas used increases, the level and the number of subordinate maximum decrease, and the field pattern takes the "ideal form" shown in Fig. 1. As a result of the experiments, the following values of power at the point of maximum were obtained:

$$
\begin{aligned}
& P_{\text {max }, 8}=0.203 \mu \mathrm{W}, \\
& P_{\text {max }, 16}=0.848 \mu \mathrm{W} .
\end{aligned}
$$

Averaged power at the periphery:

$$
\begin{aligned}
& P_{p e r, 8}=0.032 \mu \mathrm{W}, \\
& P_{p e r, 16}=0.078 \mu \mathrm{W} .
\end{aligned}
$$

In accordance with the formulas (8), (9), (11):

$$
\begin{gathered}
\frac{P_{\max , 16}}{P_{\max , 8}}=4.17 \approx\left(\frac{16}{8}\right)^{2}=4, \\
\frac{P_{p e r, 16}}{P_{p e r, 8}}=2.44 \approx \frac{16}{8}=2 .
\end{gathered}
$$



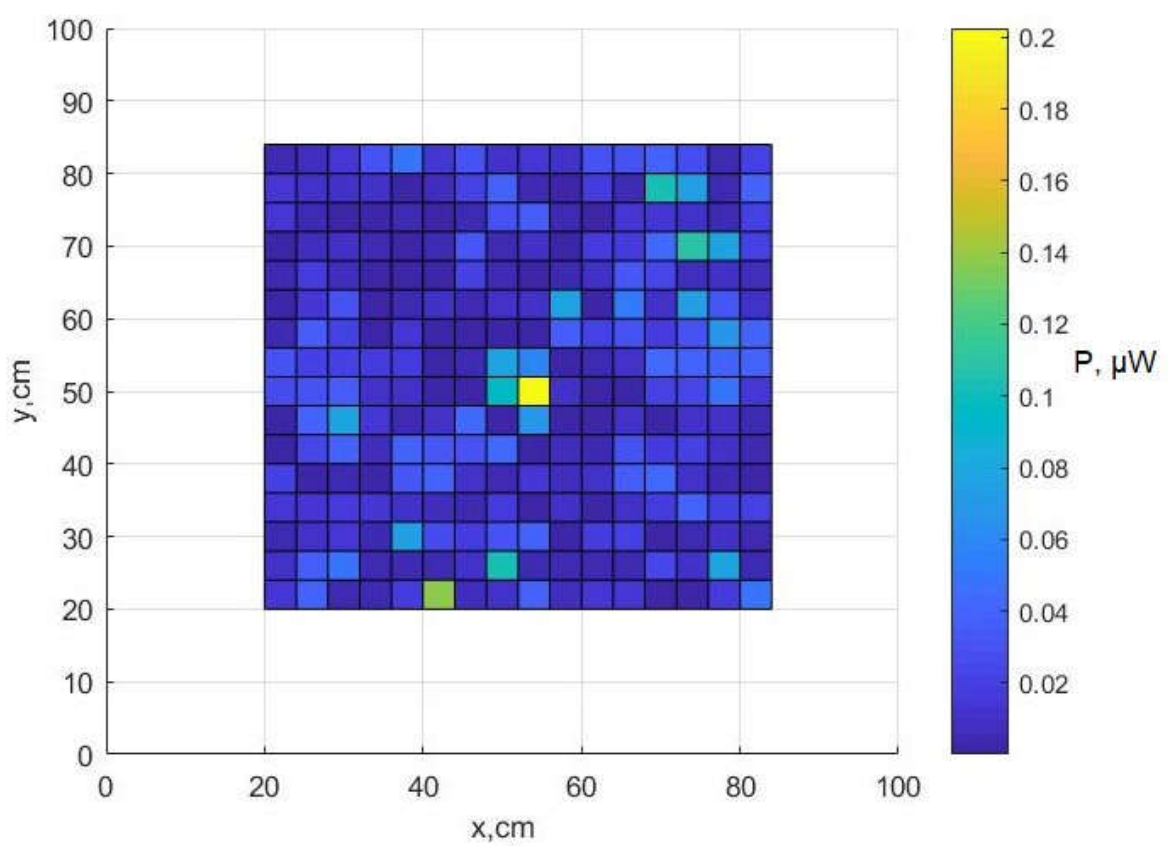

a)

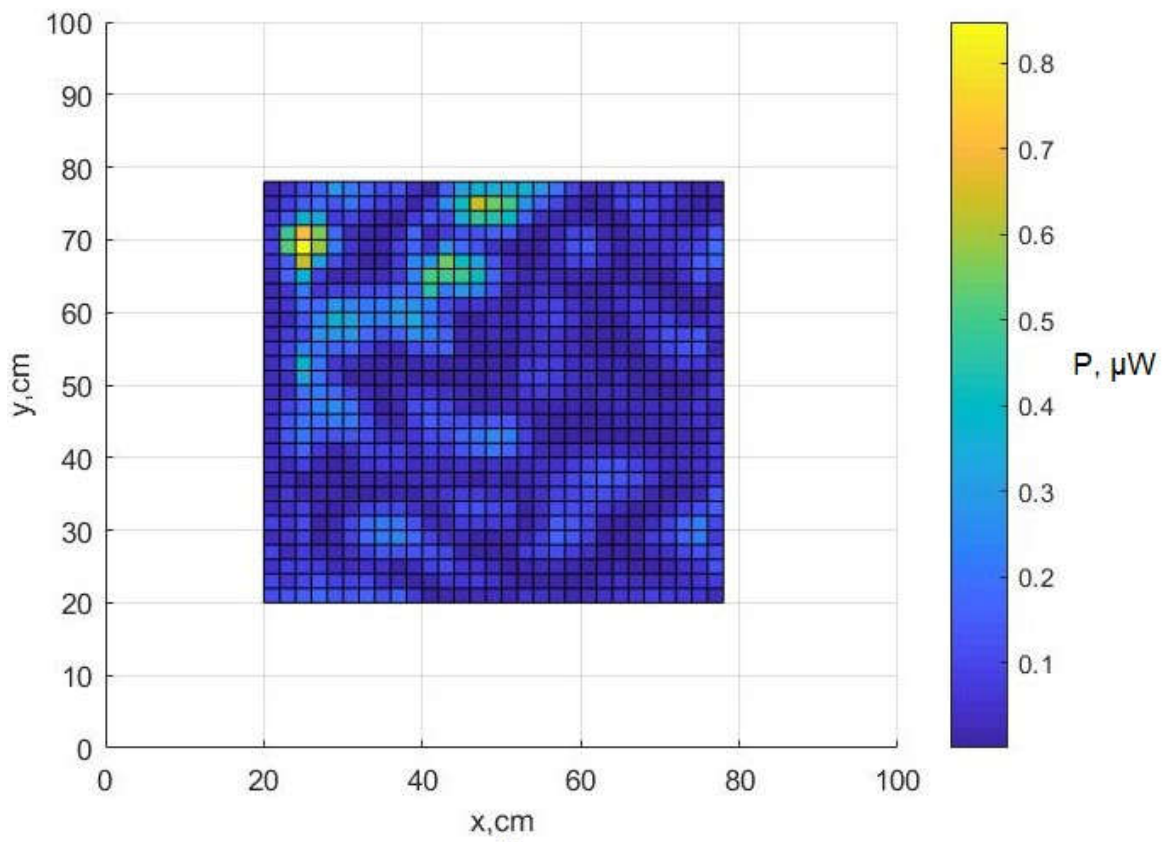

b)

Figure 8. Experimentally obtained field patterns for the case of: 8 dipole antennas $(a) ; 16$ dipole antennas $(b)$

In fact, the power at the periphery increases more slowly than the power of the maximum when the number of dipole antennas used increases. The obtained results are correlated with the estimated dependences. Thus, an important property of spotforming technology, resulting from the expression (11), is the possibility of obtaining any ratio of the power of maximum to the power at the periphery (maximum to periphery ratio) by selecting the number of dipole antennas used depending on the tasks assigned. 


\section{CONCLUSION}

We proposed Spotforming, a new approach to the spatial concentration of electromagnetic energy: at a given point, rather than in a ray, as in known approaches. This is achieved by in-phase focusing of waves at a given point.

Spotforming allows you to increase the radiation power at a given point without violating the electromagnetic compatibility with other devices in the rest of the space.

The increased power can be used to increase the speed of information transmission in communication networks, to increase the secrecy of communication systems, for wireless power transmission and for other tasks.

The authors are currently working on the application of this approach for the challenges of transferring video streams to virtual reality helmets in order to free the user from the cable between the helmet and the console.

In the long term, the implementation of the change of the location of the point of maximum in the area under consideration in real time is considered, as well as the achievement of the antinodes of the field at several points. This is a generalization of the proposed technology in the case where there are several receivers that can be mobile.

\section{REFERENCES}

1. Balanis, C. A. (2005). Antenna theory: Analysis and design (3rd ed.). Columbus, Ohio, USA : A John Wiley \& Sons.

2. Stutzman, W. L., \& Thiele, G. A. (2012). Antenna theory and design (3rd ed.). Whittemore, Iowa, USA : Courier Westford.

3. Nordrum, A., \& Clark, K. (2017, July 15). 5G bytes: beamforming explained. IEEE Spectrum. Retrieved from https://spectrum.ieee.org/video/telecom/wireless/5g-bytes-beamforming-explained.

4. Haynes, T. (1998, March 26). A primer on digital beamforming. Retrieved from http://citeseerx.ist.psu. edu/viewdoc/download?doi=10.1.1.583.5008\&rep=rep1\&type=pdf.

5. Pronin, S., Pimenov, P., \& Myrova, L. (2017). Vozdeystviye na priyemo-peredayushchiye radioelektronnyye sredstva. Raspredelennaya sistema sverkhkorotkoimpul'snogo elektromagnitnogo izlucheniya: eye komponenty i ispol'zovaniye [Impact on transceiving radio-electronic means. Distributed system of ultrashort impulse electromagnetic radiation: components and use]. Radioelektronnyye tekhnologii [Radio-Electronic Technologies], 2017(5), 69-72 (in Russian).

6. Liu, L., Zhang, R., \& Chua, K.-C. (2017). Multi-antenna wireless powered communication with energy beamforming. IEEE Transactions on Communications, 62(12), 4349-4361. doi: 10.1109/TCOMM.2014.2370035.

7. Golbon-Haghighi, M.-H. (2016). Beamforming in wireless networks. In: H. K. Bizaki (Ed.), Towards $5 G$ Wireless Networks. A Physical Layer Perspective (pp. 163-192). IntechOpen. doi: 10.5772/66399.

8. Feng, J., Chang, C.-W., Sayilir, S., Lu, Y.-H., Jung, B., Peroulis, D., \& Hu, Y. C. (2010). Energyefficient transmission for beamforming in wireless sensor networks. In: 7th Annual IEEE Communications Society Conference on Sensor, Mesh and Ad Hoc Communications and Networks (SECON) (pp. 1-9). doi: 10.1109/SECON.2010.5508256. 